\title{
BMJ Open Evidence of sociocultural factors influencing intimate partner violence among young women in sub-Saharan Africa: a scoping review
}

\author{
Maria Suzana Maguele (D) , ${ }^{1,2}$ Myra Taylor, ${ }^{2}$ Nelisiwe Khuzwayo ${ }^{3}$
}

To cite: Maguele MS, Taylor M, Khuzwayo N. Evidence of sociocultural factors influencing intimate partner violence among young women in sub-Saharan Africa: a scoping review. BMJ Open 2020;10:e040641. doi:10.1136/ bmjopen-2020-040641

- Prepublication history for this paper is available online. To view these files, please visit the journal online (http://dx.doi. org/10.1136/bmjopen-2020040641).

Received 08 June 2020 Revised 15 October 2020 Accepted 19 November 2020

Check for updates

(c) Author(s) (or their employer(s)) 2020. Re-use permitted under CC BY-NC. No commercial re-use. See rights and permissions. Published by BMJ.

${ }^{1}$ Instituto Superior de Ciencias de Saude, Maputo, Mozambique ${ }^{2}$ School of Nursing and Public Health, University of KwaZuluNatal, College of Health Sciences, Durban, South Africa

${ }^{3}$ Discipline of Rural Health, University of KwaZulu-Natal, Durban, South Africa

Correspondence to Maria Suzana Maguele; suzybata@gmail.com

\section{ABSTRACT}

Objective This study carried out a scoping review of research on intimate partner violence to determine the extent to which studies on sociocultural factors influencing intimate partner violence among young women (15-24 years) have been conducted, and how different geographical areas are represented. It also considered whether the methodologies used were sufficient to describe the risk factors, prevalence and health outcomes associated with intimate partner violence among young women.

Study design Scoping review.

Methods Online databases were used to identify studies published between 2008 and 2019. The Preferred Reporting Items for Systematic Review and Meta-Analysis guidelines by Arksey and O'Malley were used to select studies, and primary studies were assessed using the Mixed Method Appraisal Tool, V.2011. Thematic content analysis was used to summarise the findings of the scoping review.

Results The majority of publications eight $(61.5 \%)$ reported cross-sectional studies, while four (31.5\%) were qualitative studies. One of the studies $(7 \%)$ collected measured data. Overall, $13(100 \%)$ of the publications examined factors influencing intimate partner violence. Using a customised quality assessment instrument, 12 $(92.3 \%)$ of studies achieved a 'high'-quality ranking with a score of $100 \%$, and $7.7 \%$ of studies achieved an 'average' quality ranking with a score of $75 \%$.

Conclusions While the quality of the studies is generally high, researches on sociocultural factors influencing intimate partner violence among young women would benefit from a careful selection of methods and reference standards, including direct measures of the violence affecting young women. Prospective cohort studies are required linking early exposure with individual, sociocultural and community factors, and detailing the abuse experienced from childhood, adolescence and youth.

PROSPERO registration number CRD42018116463. Scoping protocol publication https://doi.org/10.1186/ s13643-019-1234-y

\section{INTRODUCTION}

Intimate partner violence (IPV) is a widespread global public health concern. ${ }^{1}$
Strengths and limitations of this study

- We conducted an exhaustive search for relevant studies from five search engines, and after that the screening of abstracts and full articles was performed using a structured tool. The degree of agreement calculations revealed no significant difference, and the mixed methods tool was applied to assess the risk of bias.

- The review limited the findings to compare risk factors specific to younger women aged 15-24 years, as data on sociocultural factors influencing intimate partner violence were mostly derived from studies using existing studies in women of reproductive age.

- The use of a cross-sectional design in the included studies and use of self-administered questionnaires in accessing the experiences of intimate partner violence runs the risk of potential bias in the studies included, in respect of the study sample selection, the recall period and in obtaining socially desirable responses.

- There was a scarcity of research evidence regarding the sociocultural factors influencing intimate partner violence among young women aged 15-24 years in the sub-Saharan African settings.

According to UNESCO (2015), 85\% of the violence against women is perpetrated by their male intimate partners. ${ }^{2}$ The WHO estimates that globally one in three women $(30 \%)$ experiences violence from their partners. ${ }^{1}$ The prevalence in young women aged 15-24 years is high, ranging from $29.4 \%$ to $31.6 \%$, while the prevalence in older women above 24 years ranges from $15.1 \%$ to $37.8 \% .^{13}$ In the sub-Saharan Africa (SSA) region which carries the most substantial burden of IPV, (36.6\% of the global estimates), the prevalence among young women aged 15-24 years, ranges from $19 \%$ to $66 \% .^{3}$ Although the data are scarce in low-income and middleincome countries, (including SSA countries) regarding the IPV in young people, where the data are available the evidence points to 
increased vulnerability to IPV among the younger groups of women compared with those older. For example, a recent study conducted in low-income and middleincome countries including SSA found that female adolescents and younger adults of 15-19 years were at higher risk of IPV when compared with older groups of women. ${ }^{4}$ This pattern was mostly observed in Namibia, Senegal, Zimbabwe, Cameroon, Sierra Leone, Congo, Zambia and Rwanda. However, different patterns regarding the higher risk of IPV in older rather than younger women were found particularly in countries outside SSA such as in Europe and Central Asia. ${ }^{4}$

Globally, the numbers of young women are increasing. Worldwide, there are about 880 million women aged 15-24 years who constitute $12 \%$ of the world population. ${ }^{5}$ Mostly they are living in developing countries, including countries from the SSA region. ${ }^{5}$

It is young women in this age group who are the population group that is mostly affected by social and economic inequalities leading them to be potentially vulnerable to violence including IPV. ${ }^{6}$ For example, the high rate of unemployment affecting this group decreases their autonomy in making important decisions about their lives. ${ }^{7}$ Around $80 \%$ of young women in SSA countries cannot decide about their own health, which limits their access to health services and therefore, to prevent IPV. ${ }^{6}$

In SSA many young women, although they may be living in their parents' households and not in cohabiting relationships, initiate sexual relationships at an early age. ${ }^{389}$ The harmful social norms and the acceptance of the dominant role of men in society also perpetuate gender inequality to the detriment of women. ${ }^{4} 10$ Young women in SSA are further affected by high-risk behaviours including risky sexual behaviour and violence, including IPV and their dating older partners increases their vulnerability to IPV. ${ }^{1112}$ Authors focusing on genderbased violence research argue that young women who are dating older men are unable to take control of their relationships. ${ }^{11} 13$ An example of this is that of young women who, if they want to use protective measures such as condoms and contraceptives, must get approval from their older partner, who are not always willing to use such protective measures. ${ }^{11}$ In addition to these risk behaviours affecting this group, various other specific and contextual risk factors including parents' and peers' influences, and the use/abuse of alcohol and drugs might influence their vulnerability to partner violence. ${ }^{314}$

The problem of IPV among young women is thus of concern and deserves immediate attention in order to mitigate such violence, since this group of women is still developing, and the negative impact of IPV is likely to compromise their lives and future well-being. ${ }^{15} 16$ The factors that influence IPV among young women are well documented in developed countries, particularly in the US settings, and this includes economic, psychological, physical and cultural factors, but there is less evidence available from SSA settings. ${ }^{17-19}$ The main challenges to the prevention of IPV among this population are therefore: first, little is known about the sociocultural factors that contribute toward IPV in young women who, although still living at home, may be in violent relationships. ${ }^{820}$ Instead, research is mainly focused on household surveys aimed at measuring the prevalence of domestic violence in adult and ever married women. ${ }^{14}$ Second, due to the community acceptance of violence and social norms of male dominance, the young women's risk of violence is often not addressed. ${ }^{410}$ Third, the policies, law enforcement, reduction and prevention strategies are more focused on domestic violence in ever married or cohabiting woman, with little attention to the circumstances of young women experiencing violent relationships. ${ }^{21}$ Understanding how these factors influence IPV in young women is necessary to better inform policymakers, health sector programmers and other relevant sectors for tailor-made interventions for prevention and reduction of IPV among young women.

This study thus, aimed to map existing evidence on sociocultural factors influencing IPV among ever partnered young women aged 15-24 years, in SSA.

IPV for young women is defined in this study as an act of physical, sexual and/or psychological/emotional threats or such harm by a current or former spouse/husband, a dating partner, an ongoing sexual partner, whether or not cohabiting, against the female partner. ${ }^{22}$

\section{METHODS}

Patient and public involvement

No patient involved.

\section{Protocol and registration}

The authors undertook a scoping review of the sociocultural factors influencing IPV among young women in SSA as part of a broader study aimed at investigating the sociocultural factors influencing IPV among young women aged 15-24 years in Maputo city, Mozambique.

A scoping review is a method undertaken to determine the value and scope of a full systematic review, and is useful to summarise and disseminate research findings, to identify research gaps and for determining the need and recommendations for future research. Scoping reviews are therefore of particular use when a body of literature has not yet been comprehensively reviewed. ${ }^{23}$

To capture a more complete range of relationships, we considered not only cohabiting young women but also ever partnered young women (young women who have ever had an intimate partner, and ever experienced partner violence). An intimate partner was defined as any male partner with whom the young woman has or ever had a romantic relationship since the age of 15 , which included having sexual activities, whether spouse/ husband, boyfriend/dating partner or ongoing sexual partner/occasional partner. ${ }^{22}$

The scoping review protocol was developed and published in BMC systematic reviews and is 
Table 1 Framework for determining the eligibility of research questions (PCC)

\begin{tabular}{ll}
\hline Criteria & Description \\
\hline Population & Women aged 15-24 years \\
Concept & $\begin{array}{l}\text { Sociocultural factors associated with IPV } \\
\text { (physical and/or sexual and/or emotional/ } \\
\text { psychological violence) and/or domestic } \\
\text { violence }\end{array}$ \\
Context & sub-Saharan Africa \\
\hline
\end{tabular}

$\mathrm{IPV}$, intimate partner violence.

available via the following link: https://doi.org/10.1186/ s13643-019-1234-y.

The review was guided by the scoping review framework. It conformed to the Preferred Reporting Items for Systematic Reviews and Meta-Analysis extension for scoping review guidelines in presenting the results of this scoping review (Arksey and O'Malley). ${ }^{24}$ Briefly, the framework involves (1) identifying the research question, (2) identifying relevant studies, (3) selection of studies, (4) charting the data, and (5) collating, summarising and reporting the results. Quality assessment of the included studies as recommended by Levac et al was also performed. ${ }^{25}$

We determined the eligibility of articles to answer our research question for a scoping review study using the Population, Concept, Context nomenclature presented in table 1.

\section{Sources of information and search strategy}

A primary search of research articles published in peerreviewed journals, review articles and grey literature was conducted from the following databases: PubMed, CINAHL with full text, MEDLINE with full text, Health Source: Nursing/Academic Edition, Google Scholar (advanced search) and Academic search complete. Reference lists of the obtained studies were also searched to identify studies that could be added to the review. The search was guided by the following keywords: "intimate partner violence", "factors influencing intimate partner violence", "socio cultural factors", "dating violence", "domestic violence", "prevalence of intimate partner violence", "young women". Boolean terms (AND and OR) were used to separate the keywords and the use of Medical Subject Headings terms was also included during the search. The search was limited to studies from SSA, that were published in any language, for the 10-year period 2008-2019.

\section{Study selection}

Studies could include older women, but to meet inclusion criteria they needed to include some women aged 15-24 years. Therefore, studies were considered eligible if they met all the following inclusion criteria:
- Studies reporting evidence of the prevalence of IPV in adult women which included women aged 15-24 years.

- Studies reporting evidence on sociocultural factors influencing IPV against women.

- To be included the studies needed to have evidence of at least one type of IPV. There should be an evidence of physical, or sexual or psychological violence or co-occurrence of two or all forms of IPV.

- Study design: quantitative, qualitative, mixed methods, randomised controlled trial, cohort study, case-control study and cross-sectional study.

However, studies were deemed ineligible if:

- Studies do not report on the outcomes of the study.

- Studies were published before 2008.

- Studies examining IPV among same-sex partners.

- Studies reporting evidence on factors influencing IPV only in women above 24 years.

- Studies were not done in SSA.

- Review articles.

Following the previously outlined stages of the study selection and guided by our eligibility criteria, first, we conducted a title screening, whereby one reviewer (MSM), screened the titles from the databases. Eligible titles for abstract screening were then exported to the EndNote Library. All the studies that did not address the research questions were excluded together with all the duplicates. The reviewer sought and obtained assistance from the UKZN library services for articles that were difficult to find. The reviewer also contacted the authors to request full copies of the included articles that were not available via the databases and the UKZN library. The final EndNote database was shared among the review team for abstract screening. At this stage, two independent reviewers screened the abstracts (MSM and NP), guided by the eligibility criteria. Discrepancies between the reviewers' responses at this stage were resolved by discussions until an agreement was reached. At the third stage, the two reviewers independently screened the full articles (MSM and NP). Discrepancies between the reviewers' responses at the full-article screening stage were resolved by involving a third reviewer (NFT). The copies of the complete articles for the eligible studies were kept for data extraction by the two reviewers (MSM and NP). Lastly, a Kappa statistics' calculation was performed to determine the degree of agreement between reviewers at the fullarticle screening by using Stata V.13 software (Stata-Corp, College Station, Texas, USA).

A flow diagram of the study selection in figure 1: The Preferred Reporting Items for Systematic Reviews and Meta-Analyses 2009 flow diagram to update screening, updated from Moher $e t a l^{26}$ shows the process involved in obtaining the eligible studies.

\section{Quality assessment}

The Mixed Method Quality Appraisal Tool (MMAT), V.2011 was used to examine the quality of articles to determine the risk of bias. ${ }^{27}$ The tool was used to investigate 


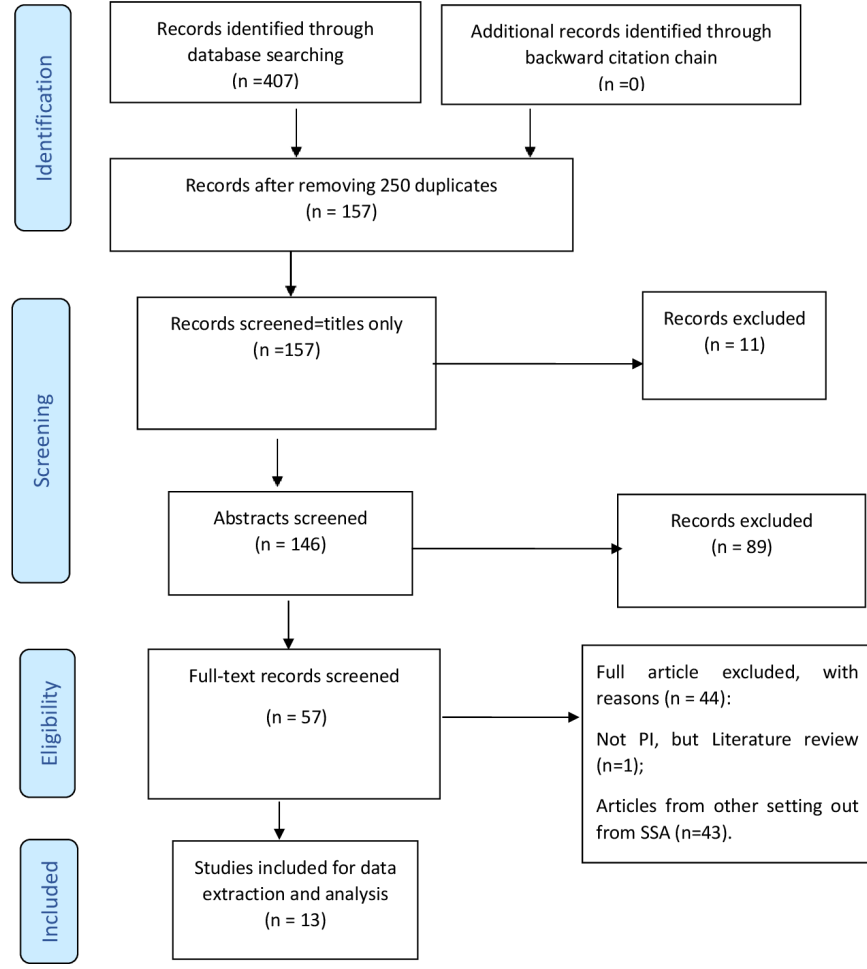

Figure 1 The Preferred Reporting Items for Systematic Reviews and Meta-Analyses 2009 flow diagram to update screening. Source: Moher et al, 2009. PI, Primary Investigation; SSA, sub-Saharan Africa.

the relationship between the theme and the research questions. Two reviewers (MSM and NP) assessed the quality of evidence of the included studies. The studies were evaluated in terms of the following domains: 'clarity of the research questions, relevant resources to address the objectives, relevant process of data analysis, the relationship between the findings and the context, and the relevance of the findings'. ${ }^{27}$ An overall quality percentage score for each of the included studies was calculated. Scores were described as low quality $(25 \%)$, fair quality $(50 \%)$, average quality $(75 \%)$ and good quality $(100 \%)$. The quality scores in this study are reported in the Results section.

\section{Data extraction}

The information addressing the research questions was thoroughly extracted using a standardised data extraction sheet from the following domains: 'author and year, study setting, population, gender, intervention, the aim of the study, study design, outcomes and key findings'.

\section{Collating and summarising the findings}

In this study, thematic analysis was found suitable for the purpose of identifying sociocultural factors influencing IPV among young women from the included studies. ${ }^{28}$ NVivo V.11 was used to extract the following relevant emergent themes: being younger than partner, education level discrepancies between partners, being married, employment and economic status of women, alcohol use by male partner, history of violence in both partners, sociocultural norms, environment and legal systems.

\section{RESULTS}

\section{Screening results}

The screening results for this scoping review are presented in figure 1. A total of 13 records were deemed eligible for data extraction and analysis. Degree of agreement was calculated after full-text screening. In respect of the full-article screening, there was $96.49 \%$ agreement versus $64.73 \%$ expected by chance between screeners, which constitutes a satisfactory agreement (Kappa statistic $=-0.90$ and $p$ value of $<0.05$ ). In addition, the McNemar's $X^{2}$ statistic indicates that there is no statistically significant difference in the proportions of yes/no answers by reviewers ( $\mathrm{p}$ value of $>0.05$ ).

\section{Characteristics of included studies}

Thirteen out of the 57 reviewed articles were eligible for data extraction. The total sample size was 13334 participants, ranging from studies with 8 to 4906 participants, with the ages ranging from 14 to 56 years. Ten of the included studies had exclusively female participants, and in three studies, there were both women and men. The women comprised 12322 participants, corresponding to $92.4 \%$ of the total sample size. The characteristics of the included studies are presented in table 2 below.

\section{Risk of bias within studies}

All 13 included studies underwent a methodological quality assessment using the MMAT V.2011. ${ }^{27}$ Twelve out of the 13 included studies were scored as high quality with a score of $100 \% .^{13171929-37}$ The remaining study had an average score of $75 \% .^{38}$ None of the included studies was scored as low quality $(25 \%)$. The overall evidence was considered to have a minimal risk of bias.

\section{Summary of the findings}

Evidence on sociocultural factors influencing IPV among young women in SSA was found in 13 studies and is presented under the following themes: being younger than partner, education level discrepancies between partners, being married, employment and economic status of women, alcohol use by male partner, history of violence in both partners, sociocultural norms, environment and legal systems.

\section{Being younger than partner}

Four studies reported that age discrepancies between women and their partners were a factor that influences IPV. The age discrepancy between partners was found to be associated with IPV both in a study conducted in South Africa among pregnant and postpartum women, ${ }^{31}$ and in a study conducted in a general population of women from rural and urban communities in Nigeria. ${ }^{29}$ In a Tanzanian study which aimed at describing and comparing the baseline prevalence of IPV among men and women, being young was associated with being both a perpetrator and 
Table 2 Summary characteristics of included articles $(n=13)$

\begin{tabular}{|c|c|}
\hline & $\begin{array}{l}\text { Number (\% of tota } \\
\text { studies) }\end{array}$ \\
\hline \multicolumn{2}{|l|}{ Publication year } \\
\hline 2008-2011 & $4(30.8)$ \\
\hline 2012-2015 & $6(46.2)$ \\
\hline 2016-2019 & $3(23)$ \\
\hline \multicolumn{2}{|l|}{ Location } \\
\hline South Africa & $2(15.4)$ \\
\hline Kenya & $3(23)$ \\
\hline Nigeria & $2(15.4)$ \\
\hline Tanzania & $2(15.4)$ \\
\hline Mali & $1(7.7)$ \\
\hline Botswana & $1(7.7)$ \\
\hline Rwanda & $1(7.7)$ \\
\hline Togo & $1(7.7)$ \\
\hline \multicolumn{2}{|l|}{ Setting: urban versus rural } \\
\hline Urban settings & $4(30.8)$ \\
\hline Rural settings & $3(23)$ \\
\hline Both urban and rural settings & $6(46.2)$ \\
\hline \multicolumn{2}{|l|}{ Setting-sector } \\
\hline Colleges & $1(7.7)$ \\
\hline Healthcare centre & $4(30.8)$ \\
\hline Households & $7(53.8)$ \\
\hline Services support centre & $1(7.7)$ \\
\hline \multicolumn{2}{|l|}{ Design } \\
\hline Cross-sectional studies & $8(61.5)$ \\
\hline Qualitative studies & $4(30.8)$ \\
\hline Longitudinal & $1(7.7)$ \\
\hline \multicolumn{2}{|l|}{ Collection of data (methods) } \\
\hline Questionnaires & $9(69.3)$ \\
\hline Interviews & $3(23)$ \\
\hline Focus group discussion & $1(7.7)$ \\
\hline \multicolumn{2}{|l|}{ Topics investigated } \\
\hline $\begin{array}{l}\text { Prevalence and factors predicting } \\
\text { IPV }\end{array}$ & $7(53.8)$ \\
\hline $\begin{array}{l}\text { Meanings and factors influencing } \\
\text { IPV }\end{array}$ & $4(30.8)$ \\
\hline Health consequences of IPV & $2(15.4)$ \\
\hline
\end{tabular}

$\mathrm{IPV}$, intimate partner violence.

a victim of violence. ${ }^{36}$ The age differences between partners were a reported predictor for IPV in a qualitative study from Botswana. ${ }^{13}$ If a young woman is married to, or in a relationship with a partner older than herself, she may struggle to air her opinions about their relationship, and further the older partner may expose the younger female partner to violence.
Education level discrepancies between partners

Findings are divergent regarding the educational level and its association with IPV across countries. For example, in a study from Togo, educated and young female partners were more likely to experience IPV. The findings suggested that the women with grade 7-10 education were 1.5-fold more likely to experience IPV compared with their counterparts with no education. ${ }^{34}$ Studies from Kenya, Tanzania and Botswana similarly suggested that a high level of education placed women at increased risk for psychological abuse. ${ }^{1735}$ In a study from Botswana, the unequal standard of knowledge between partners put young women at risk of violence, as the male partner might feel inferior and inflict violence to demonstrate that he is still superior even with a low level or without any education. In contrast, one study conducted in an urban region of Kenya aimed at evaluating the association between acceptance of IPV and IPV victimisation, suggested that young women with a high level of education were less likely to accept IPV.

\section{The women's married status}

Four studies reported marital status as a risk factor for IPV among young women. In one study, being married and having children rather than having no children influenced the young women's decision to remain in a marriage with violence. ${ }^{33}$ In one of these studies, being married was linked to the risk of IPV.$^{19}$ While the other study that reported agreement between the idea that it is the women's duty to sustain the duration of the relationship, found that this was significantly associated with acceptance of IPV. ${ }^{17}$ One study has reported that being in a formal marriage influenced young women to remain in a relationship with violence. The wedding vows taken on a legal marriage are binding for them and for them marriage is forever. ${ }^{13}$

\section{Employment and economic status of women}

Three studies reported the status of employment and low economic situation as a factor associated with IPV in young women; however, the type of violence varied according to employment status. ${ }^{13} 1736$ In a study from Tanzania aiming at describing and comparing the baseline prevalence, overlap and risk factors of psychological, physical and sexual IPV, the study findings suggested that young women who were not employed reported more IPV. ${ }^{36}$ While in a study from a rural area of Botswana which aimed at evaluating the association between acceptance of IPV and reported IPV victimisation, the study findings suggested that employed and educated young women were more likely to report psychological rather than physical abuse. ${ }^{13}$ Whereas in a study conducted in South Africa and Tanzania, reports of economic deprivation, individual level of poverty, inability to meet daily needs and living in nations with lower gross national income were predictive factors for IPV. Thus, the study's findings suggest that young women who were economically dependent or lacked sources of survival and were not owning a 
place to live were more likely to remain in a relationship with violence, since their partners were their main financial and subsistence source. ${ }^{17}$ A study conducted in a rural setting of Kenya reported that poverty and dependence were factors that hindered young women from leaving or prosecuting a violent husband, who provided the food for the family. ${ }^{35}$ There is limited research aimed at investigating economic status of young women as a risk factor for IPV in SSA setting.

\section{Alcohol use by male partner}

Three studies reported alcohol use to be associated with the risk of IPV. Alcohol use by a male partner was related to attitudes of controlling behaviour and with increased risk of IPV in young women in a study conducted in an urban area of Nigeria. ${ }^{29}$ Similarly, findings from a study by Hayes and van Baak linked alcohol abuse by a male partner to the risk of sexual and physical violence. ${ }^{32}$ The risk of IPV among those who have ever consumed alcohol was due to the negative impact of alcohol consumption, since alcohol abuse is deemed to reduce responsibility. Therefore, men use alcohol to exert power over women. In support of this, a study conducted in an urban area of Tanzania by Mulawa et al revealed that among men, having ever consumed alcohol was significantly associated with the risk of perpetrating IPV. ${ }^{36}$

\section{History of violence in both partners}

Six studies reported on previous exposure by the women to violence and IPV victimisation. The findings of these studies suggested that women who have ever been exposed to any type of violence or who have ever witnessed violence in their life were more likely to report IPV in their current relationships. One study also revealed that having a partner who has ever been involved in previous physical fights with other men was the risk factor for IPV victimisation in young women. ${ }^{32}$ Another study suggested that young women who have been involved in violence in past relationships were more likely to report IPV in their current relationships. ${ }^{19}$ One study indicated that young women who have ever perpetrated violence in a previous relationship were at higher risk to commit and to experience IPV in their current relationship. ${ }^{34}$ Three studies reported on a childhood history of violence, in that either witnessing a parent's violence or being a victim, was associated with the increased risk for IPV victimisation. ${ }^{13} 3536$

\section{Social norms}

Most of the studies in this review (8 out of 13) reported on social norms which emphasise male dominance as a risk factor for IPV. Studies linked cultural practices and social norms with increasing risk of IPV in young women. ${ }^{19} 31-353738$ Whereas attitudes to young women as subordinate and male dominance within relationship were reported in three studies. ${ }^{19} 3538$ Attitudes of young women's acceptance and their justifying violence as a husband's right were also noted in three studies, ${ }^{19} 3537$ while attitudes of men's controlling behaviour to young women were reported in one study. ${ }^{32}$ Acceptance of cultural practices such as polygamy was reported in one study. ${ }^{33}$ Practices of bride price or lobola; changing one's name and relocating to men's residence were reported in one study, ${ }^{13}$ and attitudes regarding religion commitment were reported in one study. ${ }^{34}$ The cultural context and the existing harmful social norms in SSA affect also young women and may help to explain the burden and recurrence of IPV in this setting. There is limited research aimed at investigating social norms as a risk factor for IPV among young women in SSA.

\section{Environment and legal systems}

Three studies reported on violence in the community and the political systems and the women's responses to IPV. For example, a study from Togo revealed an increasing risk of IPV in young women in communities where violence is not condemned. ${ }^{34}$ In another study aiming at investigating the lived experience of women in Botswana who had experienced emotional abuse from a partner, the findings suggested that young women who were from a specific ethnic group reported more IPV. In those communities, emotional abuse was not considered abuse as it falls under the dictates of local culture ${ }^{13}$ While studies from Tanzania and Rwanda among women who have ever experienced IPV reported on the weakness of governmental laws regarding IPV, as factors that influenced the young women's decision whether to prosecute the perpetrator or to remain in a violent relationship. ${ }^{19} 36$

\section{DISCUSSION \\ Main findings}

This study sought to map evidence of the sociocultural factors influencing IPV among young women in SSA and to identify the research gaps. The search was restricted to studies published from January 2008 to May 2019. We included in our review all papers accessing physical, sexual or psychological violence, perpetrated by an intimate male partner against the female partner. The studies could include older women as well, but to meet the inclusion criteria they needed to also include and provide data on women aged 15-24 years. Knowing that studies concerning women experiencing partner violence often use different methods and definitions to address IPV, we included in the definition of IPV the designations for women aged 15-24 years attributed by others, such as domestic violence/husband abuse/partner abuse or dating violence. Thus, the included studies used different methods, definitions, different timing/frequency and measures of IPV. For example, some studies considered women at risk of IPV to include only ever married/ cohabiting women, ${ }^{13} 323637$ other studies considered currently partnered women ${ }^{19} 3138$ and ever partnered women. ${ }^{17} 29303436$ Therefore, this discussion applied not only to cohabiting women but includes ever partnered woman who has ever had an intimate partner, and ever experienced partner violence. 
IPV occurs globally despite the actions that have been taken to prevent it in most countries. Therefore, the findings of this study have helped to underscore better the existing evidence on the sociocultural factors influencing IPV among young women in SSA. Bearing in mind the reported high prevalence and the sociocultural factors influencing the practices of IPV among young women in SSA that emerged from this review, these findings pose a global health concern regarding the need for countries to achieve the Sustainable Development Goals 5. ${ }^{39}$ Regarding this global concern, the WHO emphasises the need for research and evidence-based information to support education programmes and strategies empowering girls in skills to challenge social norms in the context of SSA where the prevalence of IPV is alarming. ${ }^{40} 41$ Moreover, a recent review aimed at evaluating what works, concerning interventions to prevent violence against girls and young women in low-income and middle-income countries, (which includes most of the countries in the SSA region), revealed the need for multilevel interventions to address young women. ${ }^{8}$ Responses should be based on community engagement to enhance their social network resources and promote women's agency, and encourage role models. The review has contributed to the required evidence-based information to provide the scientific basis needed to address sociocultural factors influencing IPV against young women in SSA. To the best of our knowledge, our study is the first scoping review of the sociocultural factors influencing IPV among young women aged 15-24 years in these settings.

It is noted that the prevalence of IPV as reported in this study differs from that from the studies from some high-resource regions, such as the USA where the overall reported prevalence of IPV in young women was not as high and was estimated at $8 \%-51.2 \% .{ }^{42-44}$ The prevalence of IPV reported in our review was much higher ranging from $28.77 \%$ to $67 \%$, and was similar to the one reported in a study conducted among young women aged 15-24 years in SSA and elsewhere, where the prevalence ranged between $19 \%$ and $66 \%{ }^{3}$ These results show that IPV among young women is common in many countries in the world but varies according to countries and regions. However, it is much higher in the SSA region, where governments are struggling to find the resources to provide effective preventive programmes to reduce IPV among young women. ${ }^{18}$ These differences in the prevalence of IPV, reported in our study, could be due to the differences in methods, differences in the effectiveness of the health services responses, differences in the health education strategies, as well as differences in the compliance with regulations and laws on violence against women and even the cultural differences within countries.

Our review reported that childhood exposure to violence, previous experience of IPV, either witnessing parents' violence or experiencing childhood violence, are risk factors for IPV. Findings from our review regarding these life course factors are also consistent with those reported in studies conducted in the USA. ${ }^{42} 45$
Further in a study by Al-Modallal, which examined the risk of partner physical violence victimisation as a function of childhood maltreatment among college women in Jordan, the findings revealed that the risk of severe physical partner violence was three times greater among women who had experienced childhood physical violence and five times greater among those who had witnessed father-to-mother violence. ${ }^{44}$ The review confirms the theoretical model which hypothesises about the relationship between the children's exposure to violence and the risk for IPV. The likelihood of experiencing IPV among women who have ever been exposed to violence in childhood might be through the mechanism of their lacking in coping skills. This may lead them to engage in violent methods when resolving conflicts, rather than non-violent conflict resolution methods. Another reason may be through the influence of their parents or their parents' modelling behaviour. Children may learn violent behaviour from their parents and might then imitate or replicate the behaviour from adulthood and across their lives. We highlighted similar findings from two studies carried out in South Africa among grade 8 learners, where the factors associated with girls' experience of IPV included childhood experiences of violence such as corporal punishment at home, school or community, and witnessing parents' violence. ${ }^{9}{ }^{46}$ These findings, therefore, highlight the importance of starting prevention efforts early in childhood, by adding in prevention strategies' programmes that may build their skills and abilities to negotiate and engage in safe relationships.

In this review, findings revealed the use of alcohol by the partner and the young age of female partner as factors that are associated with IPV. Consistent with a study by Brown et al among a clinical sample of young people aged 15-24 years, the findings revealed that physical dating violence against women was associated with poorer psychosocial functioning and the substance dependence of the partner. ${ }^{47}$ Another study by Collibee and Furman reported on alcohol use as a factor associated with the increase in dating aggression among young people. ${ }^{48}$ Kelly et al's study, which assessed the attitudes, self-efficacy and occurrence of dating violence, revealed a significant association between such violence and risk factors. These comprised the early initiation of sexual experience, drug abuse, unwillingness to engage in the initial sexual experience and inability or low self-efficacy to prevent abuse with IPV victimisation. ${ }^{49}$ Alcohol use is suggested to have an influence in reducing one's sense of responsibility and thus people engage in risky behaviours, including IPV and other forms of violence. This is in concordance with the findings from a study among adolescents' grade 8 learners in South Africa, which reports an increased risk of IPV among those adolescents using alcohol. ${ }^{950}$ It is hypothesised that the use of alcohol among men may lead them to use negative styles to resolve conflict through their limited ability to use non-violent conflict resolution methods. Moreover, men might persuade young women to engage in alcohol drinking with an expectation that 
young women will then welcome sex and then use force if they do not agree to engage in sexual activity. ${ }^{46}$ Widespread alcohol consumption and its connection with violence among young people has been in the spotlight of research in SSA and US settings. It is thus crucial to tackle alcohol use and its association with violent attitudes when implementing IPV programmes among young people and thus to teach young women to recognise and to avoid engaging in such violent relationships.

Although cultural differences exist between settings, IPV is a broad phenomenon that prevails worldwide. Our review findings reported on gender inequalities, cultural practices, and the community and legal systems associated with increased risk of IPV. ${ }^{13235}$ In support of our findings are the studies from the US settings. ${ }^{49} 51$ For example, Straus and Gozjolko, in a study which analysed 13877 university students who were in dating relationships, reported that attitudes of coercive control of women by men are associated with increased risk of IPV.$^{51}$ Similarly, the prevailing patriarchal norms of male dominance influence the relationship dynamics among the Maori women and also shape their decision of remaining in a violent relationship. ${ }^{52}$ Recent studies from Bangladesh and Vietnam highlighted persistent social norms of male dominance that are still prevailing in those societies. ${ }^{5354}$

The findings from our review emphasise that IPV remains a burden across countries and continents, especially in SSA. It appears that cultural differences between settings may explain the differences in rates, types and responses rather than the occurrence of IPV. For example, the study among grade 8 learners in South Africa reported a reduced risk of emotional violence among women who disagreed with the ideologies of male dominance. ${ }^{46}$

However, disagreeing with partners or arguing might increase the risk of physical violence among those partners who use violence to resolve conflict or those dating partners with strong ideologies of male dominance. ${ }^{9}$ Prevention programmes would need to challenge these ideologies in a safe environment and to raise awareness about non-violent ways of resolving conflicts between young partners. Moreover, a longitudinal research is needed to determine whether protective factors work in mixed or separated gender groups. Thus, the effective interventions will need to tackle empowering girls with skills to challenge negative social norms, and to tackle policies and law enforcement that condone all forms of violence against women from childhood across their lifespan.

The findings from this review have confirmed the contribution of factors at the individual, sociocultural and community levels that influence IPV among young women in SSA. This review has also provided additional evidence on the contextual sociocultural factors that may increase young women's vulnerability to IPV in the setting of SSA. The particular findings reported on cultural practices of polygamy, payment of lobola for marriage, involvement with older men, changing the name of the woman who relocates to the man's residence, and childhood experience of violence, including attitudes to child punishment, increase the current information by providing a unique context of the sociocultural factors placing young women at increased risk of IPV in SSA. These traditional practices still prevail in most countries in SSA and contribute to IPV behaviours. In contrast, sociocultural factors are less common in developed countries outside SSA such as the US setting where the researches on IPV among young women are often conducted, and the typical findings are related to whether the young women have witnessed or experienced IPV during childhood, their having multiple partners, and the use of drugs and alcohol among young people.

Given that the contextual factors which have emerged often constrain the existing strategies aimed at reducing IPV among young women in SSA, new approaches for addressing young women in SSA should be added to the current interventions. Therefore, additional efforts are necessary to increase young women's ability to challenge harmful social and cultural norms, and to build their skills to avoid their engagement with older partners and in violent relationships. There is also an urgent need for those in such relationships to enhance their ability to decide whether to remain and manage such violent relationships or to have the option to leave.

Although the research on sociocultural factors influencing IPV among young women is reported less in the SSA setting, our review is noteworthy of several contributions. This review has first contributed to the body of literature by examining, comparing and synthesising the studies' findings on the evidence of the factors influencing IPV against young women across multiple forms of IPV in SSA countries. Second, our review provided quantitative and qualitative data, regarding factors influencing IPV among young women in SSA, and this has been underlined by the rigorous standards, criteria and methodology used in this review process. This has helped to examine the emerged individual, sociocultural and community factors that show promise to guide the design of contextual and effective preventive interventions addressing young women in SSA. Finally, the review emphasises the sociocultural factors placing young women at increased vulnerability of IPV in SSA. In this setting, the majority of communities are dictated to by the social norms which give privilege to men's dominance over women, leading to gender inequalities and promoting IPV, which needs to be targeted. This synthesis is important, given the focus of the research on young women, a group that is most affected by gender inequalities resulting in higher risk for IPV. Due to the harmful social norms that still prevail in SSA and the limited research on factors influencing IPV among young women, there is still a need to provide additional research on other sociocultural factors affecting young women such as peer pressure, parental influences, socioeconomic and educational background of parents, in order to adequately contribute to effective intervention programmes to reduce IPV among young women in SSA. Such programmes to reduce IPV among this vulnerable 
population group should be initiated early, using contextual and multilevel approaches to safeguard the physical, sexual and emotional well-being of young women.

\section{Strengths and limitations}

This study is a unique scoping review to map evidence on sociocultural factors influencing IPV among young women in SSA and to provide evidence-based recommendations, a topic for which few review studies exist outside America.

The scoping review methodology employed herein was detailed. We conducted an exhaustive search for relevant studies from five search engines. The screening of abstracts and full articles was performed using a structured tool. Then the degree of agreement calculations after full-article screening revealed no significant difference between the screeners' responses. The MMAT was applied to assess the risk of bias. However, despite the above-mentioned strengths, limitations regarding the study design of the included studies were encountered. Most studies were cross-sectional in design. There was also potential for bias in the studies included in respect of their selection of the study sample and the recall period. Moreover, the evidence of IPV experiences was mainly assessed in most of the studies using self-administered questionnaires. This method runs the risk of potential recall bias in obtaining socially desirable responses. ${ }^{55} \mathrm{Few}$ studies were focused specifically on young women aged 15-24 years. Data on sociocultural factors influencing IPV among young women aged 15-24 years were mostly derived from existing studies researching IPV in women of reproductive ages, which included young women. This may have limited the findings to compare risk factors specific to young women. Thus, this highlights the need for more primary research focused on sociocultural factors influencing IPV among young women in SSA to contribute evidence-based prevention programmes to reduce IPV among this vulnerable population group.

\section{CONCLUSION}

Although unevenly distributed among SSA countries, the studies revealed considerable research evidence of the factors associated with IPV in some of these settings. Many of the studies that provided evidence about IPV among young women were carried out in the US settings, whereas few studies were from SSA. The findings point to the scarcity of research evidence regarding the sociocultural factors influencing IPV among young women in SSA. Nevertheless, IPV is a common phenomenon in SSA. It is mainly influenced by the factors interacting at the individual, community and societal levels such as young age of women, discrepancies in the education level between partners, young women's marital status, low economic/unemployment status of women, alcohol use by women's partner, history of violence including childhood violence experienced by both partners, social norms of male dominance, and environmental and legal systems.
Understanding about the sociocultural risk factors for IPV among specific groups of young women in SSA will help to design contextual preventive programmes that contribute to the reduction of their vulnerability and the trajectories of victimisation from childhood and across the life course. Thus, effective prevention programmes should incorporate actions empowering young women economically and with education to enhance their awareness and autonomy, and develop their ability to challenge harmful social norms, allowing young women to pursue their relationships' lives with integrity and free from violence.

\section{Implications for practice}

Risk factors such as young age of young women, discrepancies in the education level between partners, young women's marital status, low economic/unemployment status of young women, alcohol use by young women's partner, history of violence including childhood violence experienced by both partners, social norms of male dominance, and environmental and legal systems are associated with IPV among young women and therefore constitute a public health concern. We recommend that health promoters and providers at health system facilities, including those at community and political levels, continue monitoring and providing health assistance and political and legal support for the victims. Action is also needed to empower young women concerning their awareness about IPV in a community-based approach.

\section{Implications for research}

This scoping review shows a gap in research focusing on sociocultural factors influencing IPV among young women in SSA. The existing few studies conducted in SSA, and most of the studies undertaken in SSA setting, are cross-sectional studies. The implementation of qualitative and longitudinal studies focusing on young women would be beneficial in providing more understanding of the factors underpinning IPV and guide proper preventive interventions.

Acknowledgements The Instituto Superior de Ciências de Saúde (ISCISA)— Maputo, Mozambique, the Netherlands Initiative for Capacity Development in Higher Education (NICHE) and the University of KwaZulu-Natal (UKZN) —Durban, South Africa, are acknowledged for the provision of resources toward this review. The UKZN Systematic Review Services is acknowledged for the provision of courses on Systematic Reviews methodology and participating in the screening process. The UKZN College of Health Sciences Library services is acknowledged for the workshops on database searches and provision of resources to conduct this research. NPV is acknowledged for participation in the screening process. NFT is acknowledged for resolving discrepancies between two other screeners and for the contribution in reviewing the manuscript and for adding on valuables inputs.

Contributors MSM conceptualised and prepared the draft proposal of the study under the supervision of MT and NK. MT and NK assisted with the manuscript redaction. MSM prepared the manuscript, and MT and NK reviewed it. Respectively MSM, MT and NK contributed to the reviewed draft version of the manuscript and approved the final version.

Funding The work is part of a large study that has been conducted by MSM with support of ISCISA in partnership with the Netherlands Initiative for Capacity Development in Higher Education (NICHE) and the University of KwaZulu-Natal (UKZN) College of Health Science as part of a PhD study conducted by MSM. 
Competing interests None declared.

Patient and public involvement Patients and/or the public were not involved in the design, or conduct, or reporting, or dissemination plans of this research.

\section{Patient consent for publication Not required.}

Provenance and peer review Not commissioned; externally peer reviewed.

Data availability statement The majority of data relevant to this study are included in the article or uploaded as supplemental information.

Open access This is an open access article distributed in accordance with the Creative Commons Attribution Non Commercial (CC BY-NC 4.0) license, which permits others to distribute, remix, adapt, build upon this work non-commercially, and license their derivative works on different terms, provided the original work is properly cited, appropriate credit is given, any changes made indicated, and the use is non-commercial. See: http://creativecommons.org/licenses/by-nc/4.0/.

ORCID iD

Maria Suzana Maguele http://orcid.org/0000-0002-7870-2534

\section{REFERENCES}

1 García-Moreno C, Pallitto C, Devries K, et al. Global and regional estimates of violence against women: prevalence and health effects of intimate partner violence and non-partner sexual violence. World Health Organization, 2013.

2 UNESCO. Relatorio Anual UNESCO Mocambique, 2015.

3 Stöckl H, March L, Pallitto $\mathrm{C}$, et al. Intimate partner violence among adolescents and young women: prevalence and associated factors in nine countries: a cross-sectional study. BMC Public Health 2014;14:751.

4 Coll CVN, Ewerling F, García-Moreno C, et al. Intimate partner violence in 46 low-income and middle-income countries: an appraisal of the most vulnerable groups of women using Nationa health surveys. BMJ Glob Health 2020;5:e002208.

5 Group WB. World population prospects 2019, 2020. Available: https://dataworldbankorg/indicator/SLEMP1524SPZS?view=chart

6 MYWorld analytics. New York: United nations, 2014. Available: https://www.unaids.org/sites/default/files/media/images/gap_report popn_02_girlsyoungwomen_2014july-sept.pdf

7 Women U, UNICEF. International technical guidance on sexuality education: an evidence-informed approach. UNESCO Publishing 2018.

8 Yount KM, Krause KH, Miedema SS. Preventing gender-based violence victimization in adolescent girls in lower-income countries: systematic review of reviews. Soc Sci Med 2017;192:1-13.

9 Shamu S, Gevers A, Mahlangu BP, et al. Prevalence and risk factors for intimate partner violence among grade 8 learners in urban South Africa: baseline analysis from the Skhokho supporting success cluster randomised controlled trial. Int Health 2016;8:ihv068-26.

10 Iman'ishimwe Mukamana J, Machakanja P, Adjei NK. Trends in prevalence and correlates of intimate partner violence against women in Zimbabwe, 2005-2015. BMC Int Health Hum Rights 2020;20:2.

11 Morrell R, Jewkes R, Lindegger G. Hegemonic masculinity/ masculinities in South Africa: culture, power, and gender politics. Men Masc 2012;15:11-30.

12 Moçambique I. Inquérito Demográfico E de Saúde; 2011. back to cited text, 2011.

13 Thupayagale-Tshweneagae G, Seloilwe ES. Emotional violence among women in intimate relationships in Botswana. Issues Ment Health Nurs 2010;31:39-44.

14 Liu W, Mumford EA, Taylor BG. The relationship between parents intimate partner victimization and youths' adolescent relationship abuse. J Youth Adolesc 2018;47:321-33.

15 Grose RG, Chen JS, Roof KA, et al. Sexual and reproductive health outcomes of violence against women and girls in lower-income countries: a review of reviews. J Sex Res 2020:1-20.

16 Grose RG, Roof KA, Semenza DC, et al. Mental health, empowerment, and violence against young women in lower-income countries: a review of reviews. Aggress Violent Behav 2019;46:25-36.

17 Sabina C. Individual and national level associations between economic deprivation and partner violence among college students in 31 national settings. Aggress Behav 2013;39:247-56.

18 Mannell J, Willan S, Shahmanesh M, et al. Why interventions to prevent intimate partner violence and HIV have failed young women in southern Africa. J Int AIDS Soc 2019;22:e25380.
19 Mannell J, Jackson S, Umutoni A. Women's responses to intimate partner violence in Rwanda: rethinking agency in constrained social contexts. Glob Public Health 2016;11:65-81.

20 Cruz GV, Domingos L, Sabune A. The characteristics of the violence against women in Mozambique. Health 2014;06:1589-601.

21 McCloskey LA, Boonzaier F, Steinbrenner SY, et al. Determinants of intimate partner violence in sub-Saharan Africa: a review of prevention and intervention programs. Partner Abuse 2016;7:277-315.

22 Heise L, Hossain M. Strive technical brief: measuring intimate partner violence, 2017

23 Peters MDJ, Godfrey CM, Khalil H, et al. Guidance for conducting systematic scoping reviews. Int $J$ Evid Based Healthc 2015;13:141-6.

24 Arksey H, O'Malley L. Scoping studies: towards a methodological framework. Int J Soc Res Methodol 2005;8:19-32.

25 Levac D, Colquhoun H, O'Brien KK. Scoping studies: advancing the methodology. Implement Sci 2010;5:69.

26 Moher D, Liberati A, Tetzlaff J, et al. Reprint--preferred reporting items for systematic reviews and meta-analyses: the PRISMA statement. Phys Ther 2009;89:873-80.

27 Pluye P, Robert E, Cargo M. Proposal: a mixed methods appraisal tool for systematic mixed studies reviews. Montréal: McGill University, 2011: 2. 1-8.

28 Booth A, Noyes J, Flemming K, et al. Structured methodology review identified seven (retreat) criteria for selecting qualitative evidence synthesis approaches. J Clin Epidemiol 2018;99:41-52.

29 Balogun MO, Owoaje ET, Fawole OI. Intimate partner violence in southwestern Nigeria: are there rural-urban differences? Women Health 2012;52:627-45.

30 Abasiubong F, Abasiattai AM, Bassey EA, et al. Demographic risk factors in domestic violence among pregnant women in Uyo, a community in the niger delta region, Nigeria. Health Care Women Int 2010;31:891-901.

31 Groves AK, Moodley D, McNaughton-Reyes L, et al. Prevalence, rates and correlates of intimate partner violence among South African women during pregnancy and the postpartum period. Matern Child Health J 2015;19:487-95.

32 Hayes BE, van Baak C. Risk factors of physical and sexual abuse for women in Mali: findings from a nationally representative sample. Violence Against Women 2017;23:1361-81.

33 Makayoto LA, Omolo J, Kamweya AM, et al. Prevalence and associated factors of intimate partner violence among pregnant women attending Kisumu district Hospital, Kenya. Matern Child Health J 2013;17:441-7.

34 Moore AR. Types of violence against women and factors influencing intimate partner violence in Togo (West Africa). J Fam Violence 2008;23:777-83.

35 Mugoya GCT, Witte TH, Ernst KC. Sociocultural and victimization factors that impact attitudes toward intimate partner violence among Kenyan women. J Interpers Violence 2015;30:2851-71.

36 Mulawa M, Kajula LJ, Yamanis TJ, et al. Perpetration and victimization of intimate partner violence among young men and women in Dar ES Salaam, Tanzania. $J$ Interpers Violence 2018;33:2486-511.

37 Odero M, Hatcher AM, Bryant C, et al. Responses to and resources for intimate partner violence: qualitative findings from women men, and service providers in rural Kenya. $J$ Interpers Violence 2014;29:783-805.

38 Boonzaier FA, van Schalkwyk S. Narrative possibilities: poor women of color and the complexities of intimate partner violence. Violence Against Women 2011;17:267-86.

39 Nations U. Goal 5: achieve gender equality and empower all women and girls, 2020. Available: https://wwwunorg/ sustainabledevelopment/gender-equality/

40 Organization WH. Strengthening health systems to respond to women subjected to intimate partner violence or sexual violence: a manual for health managers, 2017.

41 Organization WH. Changing cultural and social norms that support violence, 2009.

42 Novak J, Furman W. Partner violence during adolescence and young adulthood: individual and relationship level risk factors. $J$ Youth Adolesc 2016;45:1849-61.

43 Mitra M, Mouradian VE, McKenna M. Dating violence and associated health risks among high school students with disabilities. Matern Child Health J 2013;17:1088-94.

44 Al-Modallal $\mathrm{H}$. Childhood maltreatment in college women: effect on severe physical partner violence. J Fam Violence 2016;31:607-15.

45 Tyler KA, Melander LA, Noel H. Bidirectional partner violence among homeless young adults: risk factors and outcomes. J Interpers Violence 2009;24:1014-35. 
46 Russell M, Cupp PK, Jewkes RK, et al. Intimate partner violence among adolescents in Cape town, South Africa. Prev Sci 2014;15:283-95.

47 Brown A, Cosgrave E, Killackey E, et al. The longitudinal association of adolescent dating violence with psychiatric disorders and functioning. J Interpers Violence 2009;24:1964-79.

48 Collibee C, Furman W. A Moderator model of alcohol use and dating aggression among young adults. J Youth Adolesc 2018;47:534-46.

49 Kelly PJ, Cheng A-L, Peralez-Dieckmann E, et al. Dating violence and girls in the juvenile justice system. $J$ Interpers Violence 2009;24:1536-51.

50 Viner RM, Ozer EM, Denny S, et al. Adolescence and the social determinants of health. Lancet 2012;379:1641-52.
51 Straus MA, Gozjolko KL. "Intimate Terrorism" and Gender Differences in Injury of Dating Partners by Male and Female University Students. $J$ Fam Violence 2014;29:51-65.

52 Hoeata C, Nikora LW, WW L, et al. Māori women and intimate partner violence: some sociocultural influences, 2011.

53 James-Hawkins L, Salazar K, Hennink MM, et al. Norms of masculinity and the cultural narrative of intimate partner violence among men in Vietnam. J Interpers Violence 2019;34:4421-42.

54 Yount KM, James-Hawkins L, Cheong YF, et al. Men's Perpetration of partner violence in Bangladesh: community gender norms and violence in childhood. Psychol Men Masc 2018;19:117-30.

55 Coughlin SS. Recall bias in epidemiologic studies. J Clin Epidemiol 1990;43:87-91. 